\title{
Prevalence of methicillin-resistant staphylococci isolated from different biological samples at Policlinico Umberto I of Rome: correlation with vancomycin susceptibility
}

\author{
Maria Teresa Mascellino, Alessandra Oliva, Rocco Notarnicola, Carmela Gallinelli, \\ Fernanda Chiarini \\ Department of Public Health and Infectious Diseases, Policlinico Umberto I, Rome
}

Key words: Methicillin-resistant Staphylococcus, Glycopeptides, Susceptibility

Prevalenza di stafilococchi meticillino-resistenti in differenti campioni biologici al Policlinico Umberto I di Roma: correlazione con la sensibilità alla vancomicina

\section{SUMMARY}

The methicillin-resistance is increasing all over the world in the last decade. It is more frequent among coagulase-negative staphylococci (MRCoNS); infact the $52 \%$ of S. epidermidis strains results to be resistant to methicillin. The methicillin-resistant strains also show a reduced sensitivity towards the first-line agents such as glycopeptides and other antibiotics commonly used in therapy such as trimethoprim-sulphamethoxazole, imipenem, gentamycin, fosfomycin and chlarytromicin. Unlike MRSA (Methicillin-resistant S. aureus), MRCoNS resistance to glycopeptides generally concerns teicoplanin. Although vancomycin resistance is rare in Staphylococcus isolates, the detected shift towards higher values of MICs might affect patient's clinical outcome.

\section{INTRODUCTION}

Infections due to methicillin-resistant Staphylococcus aureus (MRSA) and coagulasenegative Staphylococcus (MRCoNS) are an emerging threat worldwide both in nosocomial and community settings (5).

Italy is a country in which a median rate of MRSA infection prevalence is evident: $40 \%$ of. $S$. aureus and $80 \%$ of CoNS are methicillin-resistant (EARSS, 2008). Glycopeptides (vancomycin and teicoplanin) are the first-choice therapy against these pathogens $(7,8)$. Detection of vancomycin resistant strains remains rare. However, staphylococci with a reduced sensitivity to glycopeptides are globally spreading and represent an emergent challenge to the clinicians $(2,4)$. Infact, infections due to $S$. aureus strains with $\mathrm{MIC}>1 \mathrm{mcg} / \mathrm{ml}$ to vancomycin are associated with therapeutic failure and poor clinical outcome $(6,3)$.

Aim of the study was to analyze the prevalence of methicillin-resistance among staphylococcal strains isolated from different biological samples (blood, CVC, skin and soft tissues, purulent fluids) at Policlinico Umberto I in Rome and to evaluate the sensitivity to vancomycin correlating it with. the resistance towards methicillin and other antibiotics in the strains under study We also analyzed the phenotypic expression of PBP2a protein.

\section{MATERIALS AND METHODS}

Over a 3 months period, a total of 116 MRSA and MRCoNS isolated from blood, skin and soft tissues, biologic fluids (abscesses, milk, umbilical cord, cerebrospinal fluid, indwelling catheters) were collected and further analyzed. Catalase, coagulase and Gram-stain were used to identify the strains with subsequent speciation using the Vitek automated system (bio-Mérieux). Methicillinresistance was also analyzed by Vitek 2 .

This system detects metabolic changes by fluorescence-based methods, monitors the Kinetics of bacterial growth and calculate MICs using a unique algorithm.

E-test method was used for detecting vancomycin susceptibility. A stable gradient of 30 antibiotic concentrations on plastic strips were applied to an inoculated agar plate (Muller-Hinton agar). After incubation, an ellipse appears that intersects the MIC value scale (in ig/ml) where the concentration of the antibiotic tested inhibits microorganism growth. Rapid latex agglutination test (Oxoid) detecting PBP2a proteins in isolates of Staphylococcus, is an aid in identifying MRSA and MRCoNS. Latex particles, sensitized with a monoclonal antibody against PBP2, specifically react with methicillin-resistant staphylococci to cause agglutination visible to the unaided eye. CLSI

\section{Corresponding author: Maria Teresa Mascellino}

Public Health and Infectious Diseases, Policlinico Umberto I

Viale del Policlinico I55 - 00 I6I Rome (Italy) - Tel.: +39 0649970880 - Fax: +39 0649972628 - Cell:+39 328 I685935

E-mail: mariateresa.mascellino@uniromal.it 
breakpoints for glycopeptides MICs were used (1)

\section{RESULTS}

We analyzed 5946 different samples: 1591 showed a microbiological growth $(26.7 \%)$, of which 715 yielded Staphylococcus spp. (45\%).

The overall prevalence of staphylococcal strains was $70.9 \%$ from blood cultures, $43.8 \%$ from skin and soft tissues infections and $29.6 \%$ from biologic fluids: methicillin-resistance accounted for $28.2 \%, 9.1 \%$ and $24.4 \%$ of the total, respectively (totally 116 methicillin-resistant strains)

Among this 116 strains, 26 were S. aureus (22\%) whereas 90 were coagulase-negative staphylococci $(78 \%)$ including S. epidermidis $(52 \%)$, S. haemolyticus (15\%), S. hominis (9\%) and other Staphylococcus species (2\%). The MRSA and MRCoNS are reported in table 1. The strains more frequently involved in methicillin-resistance are the CoNS in blood cultures (especially $S$. epidermidis), followed by those in biological fluids. MRSA resulted more present in skin and soft tissues infections. $93.4 \%$ of the strains resulted positive for PBP2A agglutination test (correspondence between the results obtained by Vitek 2 system and rapid latex assay).

The overall vancomycin MIC distribution among methicillin-resistant stapylococci (MRS), performed on 97 strains, are reported in figure I. The most percentage of the tested MRS had MIC $\leq 4$ $\mu \mathrm{g} / \mathrm{ml}$ to vancomycin by E-test with a higher distribution within the range $2-4 \mu \mathrm{g} / \mathrm{ml}$ (32 isolates) and at a less extent ( 24 isolates) at $2 \mu \mathrm{g} / \mathrm{ml}$. Three strains only showed a MIC value of more than $4 \mu \mathrm{g} / \mathrm{ml}$.

None of $S$. aureus isolates was resistant to vancomycin but 2 strains showed an intermediate MIC value of $4 \mu \mathrm{g} / \mathrm{ml}$ following the CLSI breakpoints (VISA strains) (figure II a).

As far as coagulase-negative staphylococci are concerned, 3 out of 77 (3.9\%) showed low sensitivity to vancomycin (MIC between 4 and 8 $\mu \mathrm{g} / \mathrm{ml})$. All these strains belonged to $S$. epidermidis species (figure II b). These strains resulted to be also more resistant to teicoplanin, trimethoprim-sulphamethoxazole, imipenem, gentamycin, fosfomycin and chlarytromicin (data not shown).

\section{CONCLUSION}

This study confirms the high circulation of methicillin-resistant staphylococcal strains, especially in blood cultures and biological samples. A high concordance between Vitek 2 automated system and PBP2A phenotypic test has been shown in evaluating the methicillin-resistance (concordance of $93.4 \%$ ).

The methicillin-resistance is more frequent among coagulase-negative staphylococci compared to S. aureus: in fact the $52 \%$ of S. epidermidis strains results to be resistant to methicillin. Furthermore methicillin-resistant strains show a reduced sensitivity towards the first-line agents such as glycopeptides.

Although vancomycin resistance is rare in Staphylococcus isolates, E-test determination shows a shift towards higher values of MICs. This decrease of vancomycin and teicoplanin susceptibility might have important clinical implications, especially when considering the patient's clinical outcome (therapeutic failure). Moreover these strains result to be more resistant to other antimicrobial agents commonly used in therapy such as trimethoprim-sulphamethoxazole, imipenem, gentamycin, fosfomycin and chlarytromicin.

Further microbiological analyses should be necessary to evaluate the evolution of vancomycin MICs distribution in staphylococcal strains.

Table I. Prevalence of methicillin-resistance among Staphylococcal strains.

\begin{tabular}{lcc} 
& Number of strains & $(\%)$ \\
\hline S. aureus & 26 & $(22 \%)$ \\
\hline S. epidermidis & 60 & $(52 \%)$ \\
\hline S. haemolyticus & 17 & $(15 \%)$ \\
\hline S. hominis & 10 & $(9 \%)$ \\
\hline Others & 3 & $(2 \%)$ \\
\hline Total & 116 & $(100 \%)$ \\
\hline
\end{tabular}

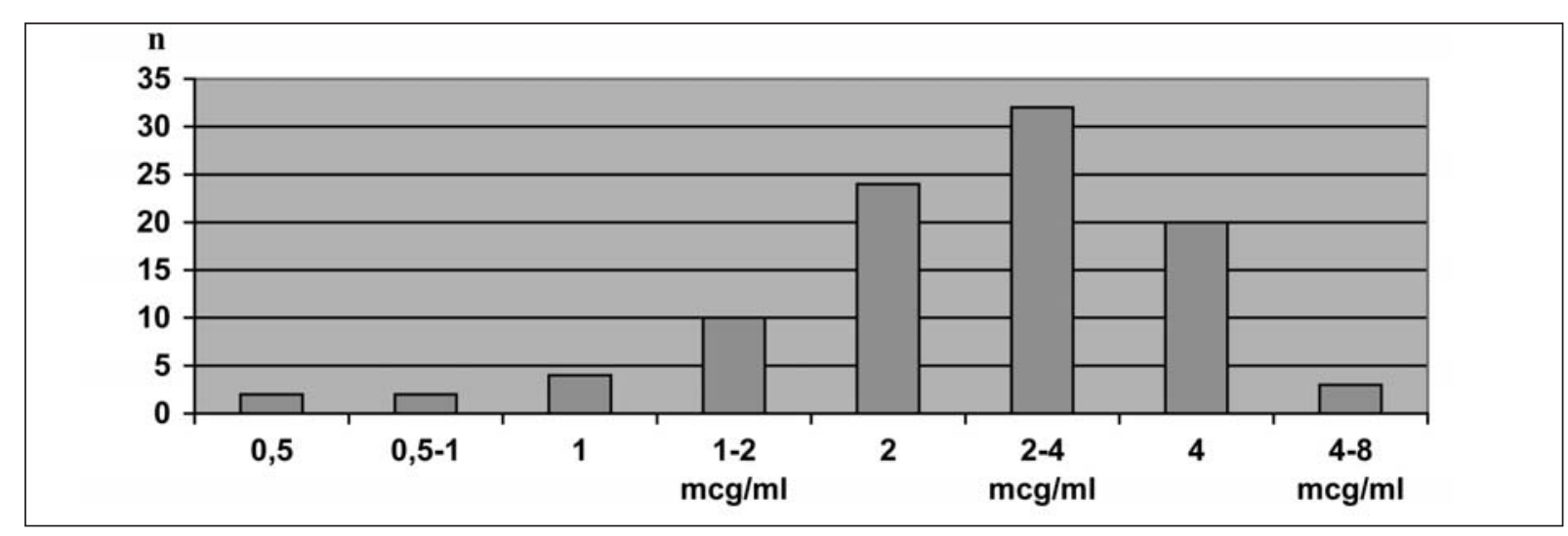

Figure I. Overall vancomycin MIC distribution among methicillin-resistant strains. 
a)

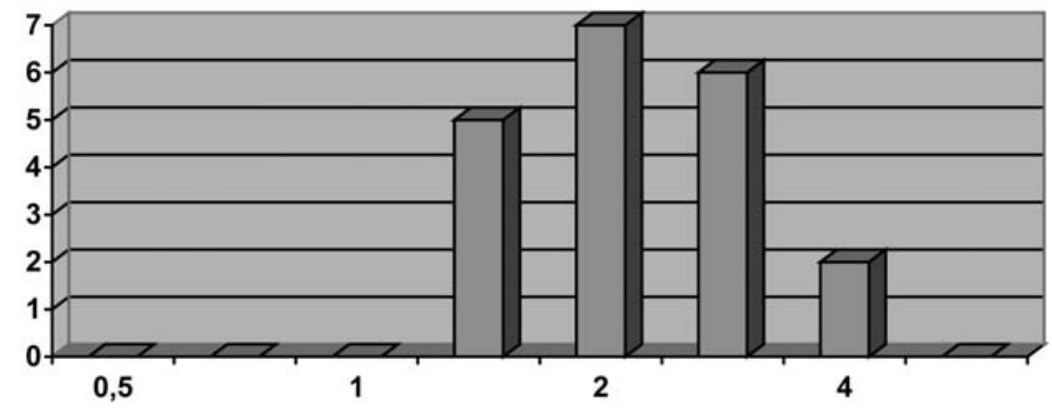

b)

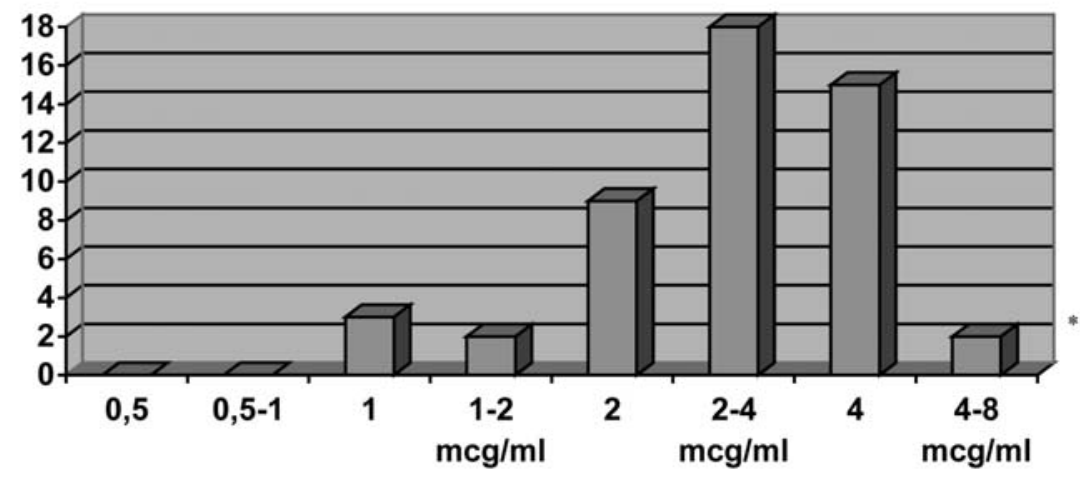

* Total strains of Methicillin-resistant Coagulase-negative Staphylococci with MIC to vancomycin, $4-8 \mathrm{mcg} / \mathrm{mL}: 3$ (2 S. epidermidis, I not identified)

Figure II. Distribution of vancomycin MICs among methicillin-resistant

S. aureus (a) and methicillin-resistant S. epidermidis (b).

\section{REFERENCES}

1. Clinical and Laboratory Standards Institute/NCCLS. Performance Standards for Antimicrobial Susceptibility Testing. Sixteenth informational supplement. M100-S16. Wayne, PA; CLSI; 2006.

2. Cremniter J, Slassi A, Quincampoix JC, et al. Decreased susceptibility to teicoplanin and vancomycin in coagulase-negative staphylococci isolated from orthopedic-device-associated infections. $J$ Clin Microbiol. 2010; 48: 1428-31.

3. Lodise TP, Graves J, Evans A, et al. Relationship between vancomycin MIC and failure among patients with methicillin-resistant Staphylococcus aureus bacteremia treated with vancomycin, Antimicrob Agents Chemother 2008; 52: 3315-20.

4. Maniati M, Petinaki E, Kontos F, et al. Rapid increase in numbers of Staphylococcus epidermidis strains with reduced susceptibility to teicoplanin. Int $J$ Antimicrob Agents 2005; 25: 346-8.

5. Manzur A, Dominguez AM, Pujol M, et al. Communityacquired methicillin-resistant Staphylococcus aureus infections: an emerging threat in Spain. Clin Microbiol Infect 2008; 14 (4): 377-80.

6. Soriano A, Marco F, Martínez JA, et al., Influence of vancomycin minimum inhibitory concentration on the treatment of methicillin-resistant Staphylococcus aureus bacteremia. Clin Infect Dis 2008; 46: 193-200.

7. Srinivasan A, Dick JD, Perl TM. Vancomycin resistance in staphylococci. Clin Microbiol Rev 2002; 15: 430-8.

8. Tacconelli E, Tumbarello $\mathrm{M}$, Donati $\mathrm{KG}$, et al. Glycopeptide resistance among coagulase-negative staphylococci that cause bacteremia: epidemiological and clinical findings from a case-control study. Clin Infect Dis 2003; 33: 1628-35. 